\title{
TRIGONOMETRIC APPROXIMATION AND UNIFORM DISTRIBUTION MODULO ONE
}

\author{
TODD COCHRANE
}

(Communicated by Larry J. Goldstein)

\begin{abstract}
We construct $n$-dimensional versions of the Beurling and Selberg majorizing and minorizing functions and use them to prove results on trigonometric approximation and to prove an $n$-dimensional version of the Erdös-Turán inequality. Finally, an application is given to counting solutions of polynomial congruences.
\end{abstract}

In this paper we construct $n$-dimensional versions of the Beurling and Selberg majorizing and minorizing functions and use them to prove a theorem on trigonometric approximation and to prove an $n$-dimensional version of the Erdös-Turán inequality. Let $e(x)=e^{2 \pi i x}, \mathbf{T}=\mathbf{R} / \mathbf{Z}, n$ be a positive integer, $\underline{x}=\left(x_{1}, \ldots, x_{n}\right)$, and $\chi_{S}$ denote the characteristic function of a set $S$. For real numbers $a_{i} \leq b_{i}$, $1 \leq i \leq n$, let $B=\prod_{i=1}^{n}\left[a_{i}, b_{i}\right]$ denote the cartesian product of the closed intervals $\left[a_{i}, b_{i}\right], \bar{B}$ denote the image of $B$ in $\mathbf{T}^{n}$, and $v(B)=\prod_{i=1}^{n}\left(b_{i}-a_{i}\right)$.

Our main result on trigonometric approximation is

THEOREM 1. For any positive integers $K_{1}, K_{2}, \ldots, K_{n}$ and any box $B=$ $\prod_{i=1}^{n}\left[a_{i}, b_{i}\right]$ with $\prod_{i=1}^{n}\left(b_{i}-a_{i}\right)<1$, there exist trigonometric polynomials

$$
T_{i}(\underline{x})=\sum_{\substack{\underline{k} \\\left|k_{j}\right| \leq K_{j}}} \alpha_{i}(\underline{k}) e(\underline{k} \cdot \underline{x}), \quad i=1,2,
$$

such that $T_{1}(\underline{x}) \leq \chi_{\bar{B}}(\underline{x}) \leq T_{2}(\underline{x})$, for $\underline{x} \in \mathbf{T}^{n}$,

$$
v(B)-\alpha_{1}(\underline{0})=\prod_{i=1}^{n}\left(b_{i}-a_{i}+\frac{2}{K_{i}+1}\right)-\prod_{i=1}^{n}\left(b_{i}-a_{i}+\frac{1}{K_{i}+1}\right),
$$

and

$$
\alpha_{2}(\underline{0})-v(B)=\prod_{i=1}^{n}\left(b_{i}-a_{i}+\frac{1}{K_{i}+1}\right)-\prod_{i=1}^{n}\left(b_{i}-a_{i}\right) .
$$

From Theorem 1 we deduce the following $n$-dimensional version of the ErdösTurán inequality.

THEOREM 2. For any positive integers $K_{1}, K_{2}, \ldots, K_{n}$, any box $B=\prod_{i=1}^{n}\left[a_{i}, b_{i}\right]$ with $v(B)<1$, and any finite set of points $\left\{\underline{x}_{1}, \underline{x}_{2}, \ldots, \underline{x}_{N}\right\}$ in $\mathbf{T}^{n}$, we have

$$
\left|\frac{1}{N} \sum_{i=1}^{N} \chi_{\bar{B}}\left(\underline{x}_{i}\right)-v(B)\right| \leq \Delta_{1}+\sum_{\substack{\underline{k} \neq \underline{0} \\\left|k_{i}\right| \leq K_{i}}}\left(\Delta_{1}+\prod_{i=1}^{n} P_{k_{i}, i}\right) \frac{1}{N}\left|\sum_{i=1}^{N} e\left(\underline{k} \cdot \underline{x}_{i}\right)\right|
$$

Received by the editors June 29, 1987.

1980 Mathematics Subject Classification (1985 Revision). Primary 10K05. 
where $\Delta_{1}$ is the quantity on the right-hand side of (1), and

$$
P_{k_{i}, i}=\min \left(\frac{1}{\pi\left|k_{i}\right|}, b_{i}-a_{i}, 1-\left(b_{i}-a_{i}\right)\right) .
$$

Comparable versions of the $n$-dimensional Erdös-Turán inequality were obtained earlier by Szüsz [5] and Koksma [4]. Szüsz showed that for any positive integer $K$, (4)

$$
\left|\frac{1}{N} \sum_{i=1}^{N} \chi_{\bar{B}}\left(\underline{x}_{i}\right)-v(B)\right| \leq c_{n}\left[\frac{1}{K+1}+\sum_{\substack{k \neq 0 \\\left|k_{i}\right| \leq K}}\left(\prod_{i=1}^{n} \frac{1}{\left|k_{i}\right|+1}\right) \frac{1}{N}\left|\sum_{i=1}^{N} e\left(\underline{k} \cdot \underline{x}_{i}\right)\right|\right]
$$

where $c_{n}$ is a constant depending only on $n$. For comparison with (3) we note that if $K_{i}=K, 1 \leq i \leq n$, then $\Delta_{1}=n /(K+1)+O_{n}\left(1 / K^{2}\right)$, where the big $O_{n}$ indicates a constant depending on $n$. Koksma's result is that for any real numbers $\lambda_{1}, \ldots, \lambda_{n}$,

$$
\left|\frac{1}{N} \sum_{i=1}^{N} \chi_{\bar{B}}\left(\underline{x}_{i}\right)-v(B)\right| \leq \Delta_{1}^{\prime}+\sum_{\substack{\underline{k} \neq \underline{0} \\\left|k_{i}\right| \leq K}} \prod_{i=1}^{n} P_{k_{i}, i}^{\prime} \frac{1}{N}\left|\sum_{i=1}^{N} e\left(\underline{k} \cdot \underline{x}_{i}\right)\right|
$$

where

$$
\begin{gathered}
\Delta_{1}^{\prime}=\prod_{i=1}^{n}\left(b_{i}-a_{i}+\frac{75}{\lambda_{i}}\right)-\prod_{i=1}^{n}\left(b_{i}-a_{i}\right), \\
K_{i}=\lambda_{i} \log \left(e \min \left(n, \lambda_{i}\right)\right), \quad 1 \leq i \leq n, \\
P_{0, i}^{\prime}=b_{i}-a_{i}+\frac{75}{\lambda_{i}}, \quad \text { and } \\
P_{k_{i}, i}^{\prime}=\min \left(b_{i}-a_{i}+\frac{75}{\lambda_{i}}, 1-\left(b_{i}-a_{i}\right)+\frac{75}{\lambda_{i}}, \frac{30}{\left|k_{i}\right|}\right), \quad k_{i} \neq 0 .
\end{gathered}
$$

All three inequalities (3), (4) and (5) are essentially of the same order of magnitude but we note that $\Delta_{1}<\Delta_{1}^{\prime}$ and that for our application below Theorem 2 yields the best result.

Inequality (3) is significant only when $\Delta_{1}<v(B)$. One way to assure this is as follows. Define

$$
f_{n}(\beta)=(1+2 \beta / n)^{n}-(1+\beta / n)^{n} \text {. }
$$

It is easy to show that $f_{1}(\beta)<1$ for $0<\beta<1$, that $f_{2}(\beta)<1$ for $0<\beta<\frac{2}{3}$ and from the inequality $f_{n}(\beta)<e^{2 \beta}-\left(1+\beta+\frac{1}{3} \beta^{2}\right)$ for $n \geq 3$, that

$$
f_{n}(\beta)<1 \text { for } 0 \leq \beta<.46, n=1,2,3, \ldots \text {. }
$$

Suppose that $\left(K_{i}+1\right)\left(b_{i}-a_{i}\right) \geq n / \beta$ for $1 \leq i \leq n$. Then $\Delta_{1} \leq f_{n}(\beta) v(B)$, and hence

$$
\Delta_{1}<v(B) \quad \text { if }\left(K_{i}+1\right)\left(b_{i}-a_{i}\right) \geq 2.2 n, \quad \text { for } 1 \leq i \leq n .
$$

Going a step further we obtain the following corollary. 
COROLlaRY. Let $\alpha, \beta$ be real numbers with $\alpha \geq 1$ and $f_{n}(\beta)<1$. Let $\left\{\underline{x}_{1}, \ldots\right.$, $\left.\underline{x}_{N}\right\}$ be a set of points in $\mathbf{T}^{n}$. Suppose that $\left|\sum_{i=1}^{N} e\left(\underline{k} \cdot \underline{x}_{i}\right)\right| \leq A$ for $\underline{k} \neq \underline{0}$, and $\left|k_{i}\right| \leq n / \beta\left(b_{i}-a_{i}\right), 1 \leq i \leq n$. Then for any box $B=\prod_{i=1}^{n}\left[a_{i}, b_{i}\right]$ such that $v(B)<1$ and

$$
v(B)>\alpha\left(1+\frac{2 n}{\beta}\right)^{n}\left(\frac{1+f_{n}(\beta)}{1-f_{n}(\beta)}\right) \frac{A}{N}
$$

we have

$$
\left|\frac{1}{N} \sum_{i=1}^{N} \chi_{B}\left(\underline{x}_{i}\right)-v(B)\right| \leq v(B)\left[f_{n}(\beta)+\frac{1}{\alpha}\left(1-f_{n}(\beta)\right)\right] .
$$

We wish to thank Hugh L. Montgomery for suggesting this problem and the method of proof. We have followed the method that Montgomery and R. C. Vaughan use to prove the one-dimensional analogues of Theorems 1 and 2 in their forthcoming book. We refer the reader to Vaaler [6] for a treatment of the onedimensional cases.

MAJORIZING AND MINORIZING FUNCTIONS. Let $H(z)$ be the entire function defined by

$$
H(z)=\left(\frac{\sin \pi z}{\pi}\right)^{2}\left[\sum_{n=-\infty}^{\infty} \frac{\operatorname{sgn}(n)}{(z-n)^{2}}+\frac{2}{z}\right]
$$

where $\operatorname{sgn}(n)=1$ or -1 according as $n$ is positive or negative, $\operatorname{sgn}(0)=0$, and $H(0)=0$. Let

Using the identity

$$
K(z)=\left(\frac{\sin \pi z}{\pi z}\right)^{2}, \quad K(0)=1
$$

$$
\left(\frac{\sin \pi z}{\pi}\right)^{2} \sum_{n=-\infty}^{\infty} \frac{1}{(z-n)^{2}}=1
$$

we can write

$$
H(z)=1-2\left(\frac{\sin \pi z}{\pi}\right)^{2}\left[\sum_{n=1}^{\infty} \frac{1}{(z+n)^{2}}+\frac{1}{2 z^{2}}-\frac{1}{z}\right],
$$

and deduce from the inequality

$$
\frac{1}{x} \geq \sum_{n=1}^{\infty} \frac{1}{(x+n)^{2}} \geq \frac{1}{x}-\frac{1}{2 x^{2}}, \text { for } x>0
$$

that

$$
\begin{aligned}
1-K(x) \leq H(x) \leq 1 & \text { for } x \geq 0 \\
-1 \leq H(x) \leq-1+K(x) & \text { for } x \leq 0
\end{aligned}
$$

The latter inequality follows from the fact that $H(z)$ is an odd function.

For any interval $[a, b]$, let

$$
V(z)=V_{[a, b]}(z)=\frac{1}{2}[H(z-a)+H(b-z)],
$$

and

$$
E(z)=E_{[a, b]}(z)=\frac{1}{2}[K(z-a)+K(b-z)] .
$$

For any function $f(\underline{x})$ defined on $\mathbf{R}^{n}$ let $\hat{f}(\underline{s})$ denote its Fourier transform $\hat{f}(\underline{s})=$ $\int_{\mathbf{R}^{n}} f(\underline{x}) e(-\underline{s} \cdot \underline{x}) d \underline{x}$. 
LEMMA 1. The functions $V(z)$ and $E(z)$ have the properties

$$
\begin{aligned}
& 0 \leq 1-E(x) \leq V(x) \leq 1 \text { for } a \leq x \leq b \\
& |V(x)| \leq E(x) \text { for } x \leq a \text { or } x \geq b
\end{aligned}
$$

(ii) $\hat{V}(s)=\hat{E}(s)=0$ for $|s| \geq 1$.

(iii) $\hat{V}(0)=b-a, \hat{E}(0)=1$.

Proof. Part (i) follows from (7). Part (ii) follows from [3, VI Theorem 7.4], using the facts that $E(z)$ and $V(z)$ are both entire functions bounded on $\mathbf{R}$ and in $L^{2}(\mathbf{R})$ (when restricted to $\mathbf{R}$ ), and are both $o\left(e^{2 \pi|\operatorname{Im} z|}\right)$. For part (iii) we note that

$$
\begin{aligned}
\int_{-\infty}^{\infty} V(x) d x & =\frac{1}{2} \lim _{t \rightarrow \infty}\left[\int_{-t-a}^{t-a} H(u) d u+\int_{b-t}^{b+t} H(u) d u\right] \\
& =-\frac{1}{2} \lim _{t \rightarrow \infty}\left[\int_{t-a}^{t+a} H(u) d u-\int_{t-b}^{t+b} H(u) d u\right] \quad \text { (since } H \text { is odd) } \\
& =b-a
\end{aligned}
$$

the latter equality following from (7). One obtains $\hat{E}(0)=1$ in a similar manner using the fact that $\int_{-\infty}^{\infty}\left(\sin ^{2} \pi x\right) / \pi^{2} x^{2}=1$.

Let $B=\prod_{i=1}^{n}\left[a_{i}, b_{i}\right]$ be a box in $\mathbf{R}^{n}$ and let $V_{i}=V_{\left[a_{i}, b_{i}\right]}\left(z_{i}\right), E_{i}=E_{\left[a_{i}, b_{i}\right]}\left(z_{i}\right)$, for $1 \leq i \leq n$. Our minorizing function for $\chi_{B}$ is defined by

$$
F_{1}(\underline{z})=F_{1}\left(z_{1}, \ldots, z_{n}\right)=\prod_{i=1}^{n} V_{i}-\prod_{i=1}^{n}\left(V_{i}+2 E_{i}\right)+\prod_{i=1}^{n}\left(V_{i}+E_{i}\right)
$$

LEMMA 2. $F_{1}(\underline{z})$ is an entire function (that is, entire in each variable), such that

(i) $F_{1}(\underline{x}) \leq \chi_{B}(\underline{x})$ for $\underline{x} \in \mathbf{R}^{n}$,

(ii) $\hat{F}_{1}(\underline{s})=0$ if $\max _{1 \leq i \leq n}\left|s_{i}\right| \geq 1$, and

(iii) $v(B)-\hat{F}_{1}(\underline{0})=\prod_{i=1}^{n}\left(b_{i}-a_{i}+2\right)-\prod_{i=1}^{n}\left(b_{i}-a_{i}+1\right)$.

ProOF. To show (i) we suppose first that $\underline{x}$ is in $B$, so that $0 \leq V_{i} \leq 1$ for $1 \leq i \leq n$. Then $F_{1}(\underline{x}) \leq \prod_{i=1}^{n} V_{i} \leq 1$. Suppose now that $\underline{x}$ is not in $B$. Without loss of generality we can assume that $x_{i} \notin\left[a_{i}, b_{i}\right]$ for $1 \leq i \leq k$ and that $x_{i} \in\left[a_{i}, b_{i}\right]$ for $k+1 \leq i \leq n$, for some $k$. By Lemma 1 (i) we have $\left|V_{i}\right| \leq E_{i}$ for $1 \leq i \leq k$, so that

$$
\prod_{i=1}^{k} E_{i} \geq \prod_{i=1}^{k} V_{i}
$$


For $1 \leq i \leq n$ we have $V_{i}+E_{i} \geq 0$, and so

$$
\begin{aligned}
\prod_{i=1}^{n}\left(V_{i}+2 E_{i}\right) & =\prod_{i=1}^{k}\left[\left(V_{i}+E_{i}\right)+E_{i}\right] \prod_{i=k+1}^{n}\left(V_{i}+2 E_{i}\right) \\
& \geq\left(\prod_{i=1}^{k}\left(V_{i}+E_{i}\right)+\prod_{i=1}^{k} E_{i}\right) \prod_{i=k+1}^{n}\left(V_{i}+2 E_{i}\right) \\
& =\prod_{i=1}^{k}\left(V_{i}+E_{i}\right) \prod_{i=k+1}^{n}\left(V_{i}+2 E_{i}\right)+\prod_{i=1}^{k} E_{i} \prod_{i=k+1}^{n}\left(V_{i}+2 E_{i}\right) \\
& \geq \prod_{i=1}^{n}\left(V_{i}+E_{i}\right)+\prod_{i=1}^{n} V_{i}, \quad \text { by }(8) .
\end{aligned}
$$

Thus $F_{1}(\underline{x}) \leq 0$.

Parts (ii) and (iii) follow from parts (ii) and (iii) of Lemma 1 and the observation that

$$
\hat{F}_{1}(\underline{s})=\prod_{i=1}^{n} \hat{V}_{i}\left(s_{i}\right)-\prod_{i=1}^{n}\left(\hat{V}_{i}+2 \hat{E}_{i}\right)\left(s_{i}\right)+\prod_{i=1}^{n}\left(\hat{V}_{i}+\hat{E}_{i}\right)\left(s_{i}\right) .
$$

Our majorizing function for $\chi_{B}$ is defined by

$$
F_{2}(\underline{z})=\prod_{i=1}^{n}\left(V_{i}+E_{i}\right)
$$

LEMMA 3. $F_{2}(\underline{z})$ is an entire function such that

(i) $F_{2}(\underline{x}) \geq \chi_{B}(\underline{x})$ for $x \in \mathbf{R}^{n}$,

(ii) $\hat{F}_{2}(\underline{s})=0$ if $\max _{1 \leq i \leq n}\left|s_{i}\right| \geq 1$, and

(iii) $\hat{F}_{2}(\underline{0})-v(B)=\prod_{i=1}^{n}\left(b_{i}-a_{i}+1\right)-\prod_{i=1}^{n}\left(b_{i}-a_{i}\right)$.

PROOF. Part (i) follows from the fact that $V_{i}+E_{i} \geq 0$ for $x_{i} \in \mathbf{R}$ and $V_{i}+E_{i} \geq 1$ for $x_{i} \in\left[a_{i}, b_{i}\right]$. Parts (ii) and (iii) follow as in the proof of Lemma 2 .

LEMMA 4. For any positive numbers $L_{1}, L_{2}, \ldots, L_{n}$ and any box $B=\prod_{i=1}^{n}\left[a_{i}, b_{i}\right]$ in $\mathbf{R}^{n}$, there exist entire functions $G_{1}(\underline{z})$ and $G_{2}(\underline{z})$ such that

(i) $G_{1}(\underline{x}) \leq \chi_{B}(\underline{x}) \leq G_{2}(\underline{x})$ for $\underline{x} \in \mathbf{R}^{n}$,

(ii) $\hat{G}_{1}(\underline{s})=\hat{G}_{2}(\underline{s})=0$ if $\left|s_{i}\right| \geq L_{i}$ for some $i, 1 \leq i \leq n$,

(iii) $v(B)-\hat{G}_{1}(\underline{0})=\prod_{i=1}^{n}\left(b_{i}-a_{i}+\frac{2}{L_{i}}\right)-\prod_{i=1}^{n}\left(b_{i}-a_{i}+\frac{1}{L_{i}}\right)$,

$$
\hat{G}_{2}(\underline{0})-v(B)=\prod_{i=1}^{n}\left(b_{i}-a_{i}+\frac{1}{L_{i}}\right)-\prod_{i=1}^{n}\left(b_{i}-a_{i}\right) \text {. }
$$

PrOOF. We simply apply Lemmas 2 and 3 to the box $\prod_{i=1}^{n}\left[L a_{i}, L b_{i}\right]$ and take $G_{i}(\underline{z})=F_{i}\left(L_{1} z_{1}, L_{2} z_{2}, \ldots, L_{n} z_{n}\right), i=1,2$.

ProOF OF THEOREM 1 . Let $G_{1}, G_{2}$ be as given in Lemma 4 with $L_{i}=K_{i}+1$, $1 \leq i \leq n$, and set

$$
T_{i}(\underline{x})=\sum_{\underline{m} \in Z^{n}} G_{i}(\underline{x}+\underline{m}), \quad i=1,2
$$


Since $G_{1}$ and $G_{2}$ are in $L^{1}\left(\mathbf{R}^{n}\right)$ (by Lemma 4(iii)), the sums for $T_{1}$ and $T_{2}$ converge almost everywhere to periodic functions modulo one with Fourier coefficients

$$
\begin{aligned}
\alpha_{i}(\underline{k}) & =\int_{0}^{1} \cdots \int_{0}^{1} T_{i}(\underline{x}) e(-\underline{k} \cdot \underline{x}) d \underline{x} \\
& =\int_{\mathbf{R}^{n}} G_{i}(\underline{x}) e(-\underline{k} \cdot \underline{x}) d \underline{x}, \quad i=1,2,
\end{aligned}
$$

that is,

$$
\alpha_{i}(\underline{k})=\hat{G}_{i}(\underline{k}), \quad i=1,2, \underline{k} \in \mathbf{Z}^{n} .
$$

Thus, by Lemma $4(\mathrm{ii}), \alpha_{i}(\underline{k})=0$ if $\left|k_{j}\right| \geq K_{j}+1$ for some $j, 1 \leq j \leq n$. By uniqueness of Fourier series we conclude that

$$
T_{i}(\underline{x})=\sum_{\substack{\underline{k} \\\left|k_{j}\right| \leq K_{j}}} \alpha_{i}(\underline{k}) e(\underline{k} \cdot \underline{x}), \quad i=1,2, \underline{x} \in \mathbf{R}^{n} .
$$

Now if $\underline{x} \in B$ modulo one, then there is a unique $\underline{m} \in \mathbf{Z}^{n}$ such that $\underline{x}+\underline{m} \in B$ and for that $\underline{m}, G_{1}(\underline{x}+\underline{m}) \leq 1 \leq G_{2}(\underline{x}+\underline{m})$. For all other integral $\underline{m}, G_{1}(\underline{x}+\underline{m}) \leq$ $0 \leq G_{2}(\underline{x}+\underline{m})$. If $\underline{x} \notin B$ modulo one then the latter inequality holds for all integral $\underline{m}$. Hence $T_{1}(\underline{x}) \leq \chi_{\bar{B}}(\underline{x}) \leq T_{2}(\underline{x})$ for all $\underline{x} \in T^{n}$. Equations (1) and (2) follow from Lemma 4(iii) and (9).

PROOF OF THEOREM 2. The function $\chi_{\bar{B}}$ has a Fourier expansion

$$
\chi_{\bar{B}}(\underline{x})=\sum_{\underline{k} \in Z^{n}} \alpha(\underline{k}) e(\underline{k} \cdot \underline{x})
$$

where $\alpha(\underline{0})=v(B)$ and

$$
|\alpha(\underline{k})|=\prod_{i=1}^{n} \frac{\left|\sin \pi\left(b_{i}-a_{i}\right) k_{i}\right|}{\pi k_{i}}, \quad \text { for } \underline{k} \neq \underline{0} .
$$

Let $T_{i}, i=1,2$, be as given in Theorem 1 . Then

$$
\left|\alpha_{i}(\underline{k})-\alpha(\underline{k})\right| \leq \int_{0}^{1} \cdots \int_{0}^{1}\left|T_{i}(\underline{x})-\chi_{\bar{B}}(\underline{x})\right| d \underline{x} \leq \Delta_{1},
$$

for $i=1,2$, and all $\underline{k}$ with $\left|k_{j}\right| \leq K_{j}, 1 \leq j \leq n$. Thus for $\underline{k} \neq \underline{0}, i=1,2$, we have

$$
\left|\alpha_{i}(\underline{k})\right| \leq \Delta_{1}+|\alpha(\underline{k})| \leq \Delta_{1}+\prod_{i=1}^{n} P_{k_{i}, i}
$$

Now

$$
\sum_{i=1}^{N} \chi_{\bar{B}}\left(\underline{x}_{i}\right) \leq \sum_{i=1}^{N} T_{2}\left(\underline{x}_{i}\right)=N \alpha_{2}(\underline{0})+\sum_{\substack{\underline{k} \neq \underline{0} \\\left|k_{i}\right| \leq K_{i}}} \alpha_{2}(\underline{k}) \sum_{i=1}^{N} e\left(\underline{k} \cdot \underline{x}_{i}\right)
$$

so that

$$
\sum_{i=1}^{N} \chi_{\bar{B}}\left(x_{i}\right)-N v(B) \leq N \Delta_{1}+\sum_{\substack{\underline{k} \neq \underline{0} \\\left|k_{i}\right| \leq K_{i}}}\left|\alpha_{2}(\underline{k})\right|\left|\sum_{i=1}^{N} e\left(\underline{k} \cdot \underline{x}_{i}\right)\right|
$$


One obtains a similar lower bound using $T_{1}$ instead of $T_{2}$. Combining these bounds with (10) yields the theorem.

PROOF OF COROLlaRY. Let $K_{i}=\left[n / \beta\left(b_{i}-a_{i}\right)\right], 1 \leq i \leq n$. Then $\Delta_{1}<$ $v(B) f_{n}(\beta)$, and using Theorem 2 with the bound $P_{k_{i}, i} \leq \bar{b}_{i}-\bar{a}_{i}, 1 \leq i \leq n$, we have

$$
\begin{aligned}
\left|\frac{1}{N} \sum_{i=1}^{N} \chi_{\bar{B}}\left(x_{i}\right)-v(B)\right| & \leq \Delta_{1}+\frac{A}{N}\left(v(B)+\Delta_{1}\right) \prod_{i=1}^{n}\left(2 K_{i}+1\right) \\
& \leq f_{n}(\beta) v(B)+\frac{A}{N}\left(1+f_{n}(\beta)\right)\left(\frac{2 n}{\beta}+1\right)^{n} \\
& \leq f_{n}(\beta) v(B)+\left(1-f_{n}(\beta)\right) \frac{v(B)}{\alpha}
\end{aligned}
$$

the latter inequality following from (6).

The corollary can be applied to polynomial congruences as follows. Let $f(\underline{x})$ be a polynomial of degree $d$ with integer coefficients, $p$ be a prime, $V$ be the set of solutions of the congruence $f(\underline{x}) \equiv 0 \bmod p$ and $\left\{\underline{x}_{i}\right\}_{i=1}^{N}=V / p=\{\underline{x} / p: \underline{x} \in V\}$. For integers $A_{i}, B_{i}$ with $0<B_{i}-A_{i} \leq p, 1 \leq i \leq n$, let $B$ be the box of integral points $\underline{x}$ with $A_{i} \leq x_{i}<B_{i}, 1 \leq i \leq n$, and $B / p=\prod_{i=1}^{n}\left[A_{i} / p, B_{i} / p\right]$. Suppose that $f(\underline{x})$ is nonsingular at infinity $\bmod p$. Then by Deligne's work [2, Theorem 3.4] on the Riemann Hypothesis for varieties over finite fields we have

$$
N=p^{n-1}(1+\theta)
$$

for some $\theta$ with $|\theta| \leq(d-1)^{n} / p^{n / 2-1}$, and

$$
\left|\sum_{i=1}^{N} e\left(\underline{k} \cdot \underline{x}_{i}\right)\right| \leq(d-1)^{n} p^{n / 2}, \quad \text { for } \underline{k} \not \equiv \underline{0} \bmod p .
$$

Applying the corollary to the box $B / p$ with $\beta=.25$ and .05 respectively, and using the bounds in (11) and (12) we have

(i) if $|B| \geq \alpha(2.22)(1+8 n)^{n}(d-1)^{n}(1+\theta)^{-1} p^{n / 2+1}$, then

$$
|V \cap B| \geq(.622)\left(1-\frac{1}{\alpha}\right) N \frac{|B|}{p^{n}}
$$

and

(ii) if $|B| \geq \alpha(1.12)(1+40 n)^{n}(d-1)^{n}(1+\theta)^{-1} p^{n / 2+1}$, then

$$
|V \cap B| \geq .947\left(1-\frac{1}{\alpha}\right) N \frac{|B|}{p^{n}} .
$$

As a comparison, we note that in the more elementary manner of $[1,(3.2)]$ one can show that if $|B| \geq \alpha 2^{n}(d-1)^{n} p^{n / 2+1}$ then

$$
|V \cap B| \geq \frac{1}{2^{n}}\left(1-\frac{1}{\alpha}\right)(1+\theta)^{-1} N \frac{|B|}{p^{n}} .
$$

Inequality (15) is not as strong as (13) or (14) but it is valid for boxes of slightly smaller cardinality. 


\section{REFERENCES}

1. T. Cochrane, The distribution of solutions to equations over finite fields, Trans. Amer. Math. Soc. 293 (1986), 819-826.

2. P. Deligne, La conjecture de Weil. I, Publ. Math. Inst. Hautes Études Sci. 43 (1974), 273-307.

3. Y. Katznelson, An introduction to harmonic analysis, Dover, New York, 1976.

4. J. F. Koksma, Some theorems on diophantine inequalities, Math. Centrum Amsterdam, Scriptum no. 5, 1950, pp. i+51.

5. P. Szüsz, Über ein Problem der Gleichverteilung, Comptes Rendus du Premier Congres des Mathematiciens Hongrois, 1950, pp. 461-472.

6. J. D. Vaaler, Some extremal functions in Fourier analysis, Bull. Amer. Math. Soc. 12 (1985), 183-216.

Department of Mathematics, Kansas State University, Manhattan, Kansas 66506 